Gustavo Fonseca de Albuquerque

Souza'

@ehttps://orcid.org/0000-0002-1794-701X

Alex Sandro Rolland Souza $a^{1,2,3}$

@ehtps://orcid.org/0000-0001-7039-2052

Gabriella de Almeida Figueredo

Praciano

CDhttps://orcid.org/0000-0002-9746-8536

Esther Soraya Lima de França'

Ohttps://orcid.org/0000-0002-8243-8115

Cinthia Freire Carvalho²

O Chttps://orcid.org/0000-0002-6670-7750

Sérgio de Sá Leitão Paiva Júnior ${ }^{4}$ ○ https://orid.org/0000-0002-9938-6673

Manuela Barbosa Rodrigues de

Souza'

C. https://orcid.org/0000-0002-7773-100X

Nadja Maria Jorge Asann0 $0^{1,3}$

(1)https://orid.org/0000-0003-3644-7333

\section{Apego materno-fetal e transtornos psiquiátricos em gestantes com fetos malformados}

\author{
Maternal-fetal attachment and psychiatric disorders in \\ pregnant women with malformed fetuses
}

DOI: $10.1590 / 0047-2085000000339$

\section{RESUMO}

Objetivo: Determinar a prevalência e os fatores associados aos sintomas de ansiedade e depressão e ao apego materno-fetal em gestantes com diagnóstico de malformações congênitas. Métodos: Estudo prospectivo de corte transversal realizado durante o período de dezembro/2019 a março/2020. Foram incluídas 77 gestantes com diagnóstico de malformação fetal atendidas no Instituto de Medicina Integral Prof. Fernando Figueira (IMIP) e excluídas aquelas $<18$ anos e as que sabiam o diagnóstico da malformação há menos de três semanas. Aplicou-se um questionário com variáveis sociodemográficas e clínicas, além da Escala Hospitalar de Ansiedade e Depressão e da Escala de Apego Materno-Fetal. Para análise estatística, foi aplicado o modelo de regressão logística multivariado com nível de significância de 5\%. Resultados: Entre as gestantes, 46,8\% possuíam sintomas ansiosos e 39\%, depressivos, sendo o apego materno-fetal médio em 54,5\% e alto em 45,5\%. Antecedentes de ansiedade e depressão e não possuir religião foram associados a maior risco de sintomas de ansiedade e depressão, e saber da malformação há $\geq 10$ semanas associou-se apenas ao risco de ansiedade e ter gestação múltipla associou-se apenas ao risco de depressão. O apego materno-fetal não foi associado a ansiedade ou depressão. Conclusão: Observou-se alta prevalência de sintomas ansiosos e depressivos em gestantes com fetos malformados, além da presença de apego materno-fetal médio/alto em todas pacientes, porém sem associação com os transtornos psiquiátricos estudados. Diante disso, urge a necessidade da criação de novas linhas de cuidado voltadas à saúde mental dessas mulheres.

\section{PALAVRAS-CHAVE}

Apego materno-fetal, sintomas depressivos, transtornos ansiosos.

\section{ABSTRACT}

Objective: To determine the prevalence and factors associated with anxiety and depression symptoms and maternal-fetal attachment in pregnant women diagnosed with congenital malformations. Methods: Prospective cross-sectional study conducted from December/2019 to March/2020. Seventy-seven managers with a diagnosis of fetal malformation attended at the Instituto de Medicina Integral Prof. Fernando Figueira (IMIP) and those under 18 years of age who did not know the diagnosis of malformation in less than 3 weeks were excluded. Applicable to a questionnaire with clinical and sociodemographic variables, in addition to the Hospital Anxiety and Depression Scale and Maternal-Fetal Attachment Scale. For statistical analysis, the multivariate logistic regression model was applied with a 5\% significance level. Results: Among pregnant women, 46.8\% had anxiety symptoms and 39\% depressive symptoms, with average maternal-fetal attachment at 54.5\% and high at $45.5 \%$. History of anxiety and depression and cannot use religion have been associated with an increased risk of anxiety and depression, whereas malformation saber for $\geq 10$ weeks, associated only with risk of anxiety and multiple pregnancy only in depression. Maternal-fetal attachment was not associated with anxiety or depression. Conclusion: If there is a high prevalence of anxious and depressive symptoms in pregnant women with malformed fetuses, in addition to the presence of medium maternal-fetal/high attachment in all patients, with no association with the studied psychiatric disorders. Therefore, request the creation of new lines of care aimed at the mental health of these women.

\section{KEYWORDS}

Maternal-fetal attachment, depressive symptoms, anxiety disorders.
Received in: 0ct/19/2020. Approved in: May/12/2021

1 Universidade Católica de Pernambuco, Recife, PE, Brasil.

2 Instituto de Medicina Prof. Fernando Figueira, Recife, PE, Brasil.

3 Universidade Federal de Pernambuco, Recife, PE, Brasil.

4 Universidade Federal Rural de Pernambuco, Recife, PE, Brasil.

Address for correspondence: Nadja Maria Jorge Asano. Rua do Príncipe, 526, Boa Vista - 50050-900 - Recife, PE, Brasil. E-mail: nadjaasano@hotmail.com 


\section{INTRODUÇÃO}

A gestação é considerada um processo fisiológico de desenvolvimento do embrião/feto que acarreta alterações importantes no corpo da mulher, mediante modificações hormonais e emocionais ${ }^{1}$. As múltiplas mudanças vivenciadas nesse período exigem adaptações e podem representar riscos para o desenvolvimento de diversas psicopatologias, como ansiedade e depressão. Essa vulnerabilidade psicológica pode interferir na interação e no vínculo que a gestante estabelece com o feto no período pré-natal, principalmente em gestantes com fetos malformados, grupo no qual se observou uma prevalência de 45,5\% e 30,3\% de sinais e sintomas clínicos de ansiedade e depressão, respectivamente ${ }^{2}$.

A relação da mãe com seu filho tem início no período pré-natal, pelas expectativas com a criança que está por vir³. Em concordância, inicia-se o apego materno-fetal, definido como a intensidade com que as mulheres se engajam em comportamentos que representam uma afiliação e uma interação com sua criança que vai nascer ${ }^{4}$. Características de personalidade são atribuídas aos bebês; as mães geralmente escolhem um nome, imaginam características físicas e gostos e estabelecem uma relação importante ${ }^{3}$. Ao receberem um prognóstico fetal ruim, os pais parecem perder a autoconfiança em como cuidar do filho e apresentam sentimentos de desesperança, falta de controle e fantasias de morte e ressurreição ${ }^{5}$.

Ademais, apresentar uma condição que oferece risco à gravidez pode levar a gestante a considerar-se inferior às outras mulheres, e isso afeta sua autoestima, que, quando baixa, pode influenciar a qualidade da ligação afetiva com o feto ${ }^{5}$. Por isso, são diversos os transtornos e consequências que a notícia da malformação fetal acarreta para a família, alterando, significativamente, toda a dinâmica familiar ${ }^{6}$. O luto vivido pela perda da possibilidade da gestação ideal pode gerar grande sofrimento psíquico, o qual se intensifica quando o apoio recebido não é suficiente diante das necessidades, podendo, em alguns casos, desencadear transtornos mentais, em especial a depressão ${ }^{7}$, além da perda do apego materno-fetal ${ }^{5}$.

Dessa forma, o nosso objetivo foi determinar a prevalência e os fatores associados aos sintomas de ansiedade e depressão e ao apego materno-fetal em gestantes com diagnóstico de malformações congênitas.

\section{MÉTODOS}

\section{Desenho do estudo e características dos participantes}

Foi realizado um estudo prospectivo de corte transversal, sendo incluídas gestantes admitidas no ambulatório de pré-natal de medicina fetal e no setor de gestação de alto risco do Instituto de Medicina Integral Prof. Fernando Figueira (IMIP), no período dezembro de 2019 a março de 2020, com diagnóstico de malformação fetal. Excluíram-se as gestantes menores de 18 anos de idade e as que não tinham conhecimento da malformação há no mínimo três semanas .

\section{Variáveis e procedimentos}

A amostra foi realizada por conveniência e todas as pacientes foram submetidas a ultrassonografia morfológica no dia da consulta na instituição por especialista em medicina fetal para confirmação ou ratificação das malformações fetais.

A coleta dos dados foi feita por pesquisadores devidamente treinados, com duração média de 25 minutos. O instrumento aplicado foi constituído de três seções. A primeira era formada pelas variáveis independentes, como idade (1830 ou > 30 anos), possuir ou não religião e companheiro, naturalidade e procedência da região metropolitana do Recife ou de outras regiões, escolaridade (1-11 ou > 12 anos), renda familiar per capita ( $<1$ ou $\geq 1$ salário mínimo), raça/cor (negra ou outras), paridade (zero ou $\geq 1$ ), número de abortos anteriores (zero ou $\geq 1$ ), idade gestacional no momento da consulta (segundo ou terceiro trimestre), idade gestacional no diagnóstico (primeiro, segundo ou terceiro trimestre), tempo de conhecimento da malformação ( $<10$ ou $\geq 10$ semanas), paciente internada, gestação planejada, gestação múltipla, trabalho remunerado, afastada pela previdência, apoio familiar, presença de doença materna associada, diagnóstico anterior de transtorno psiquiátrico, tratamento para ansiedade/depressão, acompanhamento por enfermeiro e obstetra, antecedente pessoal de malformação fetal e/ou materna, antecedente pessoal de depressão pós-parto em gestação anterior, uso de álcool na gravidez, início do uso do álcool na gravidez, uso de cigarro na gravidez, início do uso do cigarro na gravidez, uso de drogas ilícitas e início do uso de drogas ilícitas na gravidez, além da Escala de Apego Materno-Fetal - MFAS (média ou alta).

Foram avaliadas ainda variáveis relacionadas às malformações fetais, como o tipo de malformação fetal (cardiovascular, nervosa, geniturinária ou outras), incompatibilidade com a vida, prognóstico ruim, algum tratamento pós-natal e alta possibilidade de sobreviver, as quais foram baseadas na literatura e segundo um consenso de dois médicos especialistas.

A segunda seção foi realizada pela MFAS 5 , a qual foi validada para amostra brasileira ${ }^{9}$ com o objetivo de avaliar o grau de afetividade entre a gestante e o feto malformado. A escala é constituída por 24 questões, valendo de um a cinco pontos cada, de maneira que: 1 significa nunca; 2, raramente; 3 , às vezes; 4, frequentemente; e 5, quase sempre. Quanto maior a pontuação, maior o nível de apego, sendo a pontuação mínima de 24 e a máxima de 120. Para a análise, o apego materno-fetal foi delimitado como baixo (24 a 47 pontos), médio (48 a 97 pontos) e alto apego (98 a 120 pontos) ${ }^{9}$. 
A terceira seção foi composta pelo instrumento que avaliou os sinais e sintomas clínicos de ansiedade e depressão, pela Escala Hospitalar de Ansiedade e Depressão (EHAD), traduzida e validada para o Brasil ${ }^{10}$. Essa escala consiste em um instrumento de autopreenchimento contendo 14 questões de múltipla escolha, composta de duas subescalas intercaladas, para definição das variáveis dependentes: ansiedade-estado (sete questões) e depressão-estado (sete questões). Os escores da EHAD variam de zero a 21 pontos, sendo considerados os sujeitos com escores menores que 8 com ausência de sintomas clínicos para ansiedade e/ou depressão, os com escores $\geq 8$ e $\leq 10$ com sintomas leves, os com escores $\geq 11$ e $\leq 14$ com sintomatologia moderada e os com escores $\geq 15$ e $\leq 21$ com sintomas graves de ansiedade e/ou depressão ${ }^{10}$.

\section{Análise de dados}

A análise estatística foi realizada pelo software Epi-Info 7.2.4.0 (Centers for Disease Control and Prevention [CDC], Atlanta, DC). Para as variáveis categóricas, foram construídas tabelas de distribuição de frequência, e para as variáveis numéricas, medidas de tendência central (média e mediana) e dispersão (desvio-padrão [DP] e intervalo interquartil [IIQ]) foram calculadas. Em relação à análise univariada, para avaliar a associação entre as variáveis dependentes e independentes, foram utilizados os testes chi-quadrado e exato de Fisher, quando pertinentes. Para determinar a força da associação, foram calculados a razão de prevalência (RP) e seu intervalo de confiança a 95\% (IC 95\%). Além disso, realizou-se a análise multivariada, com o modelo inicial sendo composto pelas variáveis explanatórias que apresentaram valor $p<0,20$ na análise univariada, permanecendo no modelo final as variáveis com nível de significância menor que 0,05, sendo calculada a razão de odds ratio $(\mathrm{OR})$ inicial e ajustado, além de seu IC 95\%. Foi realizada, ainda, correlação linear de Pearson entre a MFAS e as subescalas de ansiedade e depressão, considerando $r=0,10$ até 0,30 (fraco); $r=0,40$ até 0,6 (moderado); $r=0,70$ até 1 (forte) ${ }^{11}$.

\section{Considerações éticas}

As pacientes candidatas ao estudo foram informadas sobre a pesquisa e convidadas a participar do estudo e, caso concordassem, foi solicitada a assinatura do Termo de Consentimento Livre e Esclarecido (TCLE). O estudo foi aprovado pelo comitê de ética em pesquisas em seres humanos do IMIP (CAAE: 67779417.3.0000.5206), sob parecer no 3.748.150, e da Universidade Católica de Pernambuco - UNICAP (CAAE: 67779417.3.0000.5206), sob parecer nº 2.310.322.

\section{RESULTADOS}

Foram abordadas 85 gestantes, entre as quais cinco foram excluídas por possuírem menos de 18 anos e três por não terem conhecimento da malformação fetal há mais de três semanas, restando, portanto, 77 gestantes com fetos malformados. Nenhuma paciente se recusou a participar da presente pesquisa.

Em relação à caracterização das gestantes incluídas, observou-se uma média de idade de 26 (DP 6,6) anos, da idade gestacional em que descobriu a malformação fetal, de 21,7 (DP 5,1) semanas e do tempo do diagnóstico, de 8,8 (DP 4,9) semanas. Destaca-se que prevaleceram as malformações do sistema geniturinário (18 - 23,4\%) (Tabela 1).

A prevalência dos sintomas de ansiedade foi de 36 (46,8\%), sendo $11(14,3 \%)$ graves, enquanto a prevalência de sintomas de depressão foi de 30 (39,0\%), sendo apenas $1(1,3 \%)$ grave. Observou-se, ainda, que nenhuma gestante apresentou apego materno-fetal leve, enquanto 54,5\% ( $n=$ 42) apresentaram apego médio (Tabela 2).

Tabela 1. Características das gestantes com fetos malformados $(n=77)$

\begin{tabular}{|c|c|c|}
\hline \multicolumn{3}{|l|}{ Variáveis } \\
\hline Idade, anos (média \pm DP) & 26,9 & 6,6 \\
\hline $18-20(n ; \%)$ & 14 & 18,2 \\
\hline $21-30(n ; \%)$ & 39 & 50,6 \\
\hline $31-41(n ; \%)$ & 24 & 31,2 \\
\hline \multicolumn{3}{|l|}{ Raça (autodeclarada) } \\
\hline Branca $(n ; \%)$ & 11 & 14,3 \\
\hline Parda (n;\%) & 46 & 59,7 \\
\hline Negra $(n ; \%)$ & 20 & 26,0 \\
\hline \multicolumn{3}{|l|}{ Escolaridade } \\
\hline Ensino fundamental 1 (1-5 anos); (n;\%) & 1 & 1,3 \\
\hline Ensino fundamental 2 (6-9 anos); (n;\%) & 19 & 24,7 \\
\hline Ensino médio (10-12 anos); (n;\%) & 44 & 57,1 \\
\hline Ensino superior (>12 anos); (n;\%) & 13 & 16,9 \\
\hline \multicolumn{3}{|l|}{ Paciente internada } \\
\hline $\operatorname{Sim}(n ; \%)$ & 12 & 15,6 \\
\hline Não (n;\%) & 65 & 84,4 \\
\hline \multicolumn{3}{|l|}{ Antecedentes de transtornos psiquiátricos } \\
\hline $\operatorname{Sim}(n ; \%)$ & 8 & 10,4 \\
\hline Não (n;\%) & 69 & 89,6 \\
\hline Paridade (mediana; ॥Q) & 1,0 & $0,0-1,0$ \\
\hline Nulípara (n;\%) & 44 & 57,1 \\
\hline Primípara (n;\%) & 23 & 29,9 \\
\hline Multípara (n;\%) & 10 & 13,0 \\
\hline Idade gestacional na consulta (média \pm DP) & 30,4 & 4,7 \\
\hline $1^{0}$ trimestre $(n ; \%)$ & 0 & 0,0 \\
\hline $2^{0}$ trimestre $(n ; \%)$ & 26 & 33,8 \\
\hline $3^{0}$ trimestre $(n ; \%)$ & 51 & 66,2 \\
\hline $\begin{array}{l}\text { Idade gestacional no diagnóstico da malformação (média } \\
\pm \text { DP) }\end{array}$ & 21,7 & 5,1 \\
\hline $1^{0}$ trimestre $(n ; \%)$ & 9 & 11,7 \\
\hline $2^{0}$ trimestre $(n ; \%)$ & 60 & 77,9 \\
\hline $3^{0}$ trimestre $(n ; \%)$ & 8 & 10,4 \\
\hline
\end{tabular}




\begin{tabular}{lcc}
\hline Variáveis & & \\
\hline Malformação fetal & 12 & 15,6 \\
Sistema cardiovascular (n;\%) & 15 & 19,4 \\
Sistema nervoso (n;\%) & 18 & 23,4 \\
Sistema geniturinário (n;\%) & 5 & 6,5 \\
Parede abdominal e trato gastrointestinal (n;\%) & 4 & 5,2 \\
Músculo esquelético (n;\%) & 6 & 7,8 \\
Cromossomopatias (n;\%) & 8 & 10,4 \\
Múltiplas malformações (n;\%) & 9 & 11,7 \\
Outros (n;\%) & & \\
Incompatível com a vida & 17 & 22,1 \\
Sim (n;\%) & 60 & 77,9 \\
Não (n;\%) & & \\
Tratamento pós-natal & 52 & 67,5 \\
Sim (n;\%) & 25 & 32,5 \\
Não (n;\%)
\end{tabular}

Tabela 2. Prevalência de ansiedade e depressão e avaliação do apego materno-fetal nas gestantes com fetos malformados $(\mathrm{N}=77)$

\begin{tabular}{|c|c|c|}
\hline Variáveis & & \\
\hline Ansiedade (média \pm DP) & 8,4 & 5,0 \\
\hline Sem sinais/sintomas clínicos (n;\%) & 41 & 53,2 \\
\hline Leve $(n ; \%)$ & 12 & 15,6 \\
\hline Moderada (n;\%) & 13 & 16,9 \\
\hline Grave (n;\%) & 11 & 14,3 \\
\hline Depressão (média \pm DP) & 6,5 & 3,9 \\
\hline Sem sinais/sintomas clínicos $(n ; \%)$ & 47 & 61,0 \\
\hline Leve $(n ; \%)$ & 18 & 23,4 \\
\hline Moderada (n;\%) & 11 & 14,3 \\
\hline Grave $(n ; \%)$ & 1 & 1,3 \\
\hline Apego materno-fetal (média \pm DP) & 96,0 & 13,8 \\
\hline Leve $(n ; \%)$ & 0 & 0,0 \\
\hline Médio (n;\%) & 42 & 54,5 \\
\hline Alto (n;\%) & 35 & 45,5 \\
\hline
\end{tabular}

Após análise multivariada, mantiveram-se com associação à ansiedade: não possuir religião (OR: 3,3; IC 95\%: 1,010,$7 ; p=0,04$ ), saber da malformação fetal $\geq 10$ semanas (OR: 0,3; IC 95\%: 0,09-0,81; $p=0,01$ ) e apresentar antecedente pessoal de ansiedade e depressão (OR: 15,2; IC 95\%: 1,61-142,65; $p=0,01$ ) (Tabela 3), enquanto, em relação à depressão, permaneceram com associação: não possuir religião (OR: 6,4; IC 95\%: 1,87-21,86; $p=0,003$ ), gestação múltipla (OR: 5,4; IC 95\%: $1,08-26,8 ; \quad p=0,03$ ) e antecedente pessoal de ansiedade e depressão (OR: 26,7; IC 95\%: 2,88-246,8; $p=0,003$ ) (Tabela 4).

Quanto ao apego materno-fetal, dividindo-se em médio e alto, após a análise multivariada, continuaram associados ao apego materno fetal médio: escolaridade entre 1-11 anos (OR: 0,21; IC 95\%: 0,05-0,82; $p=0,02$ ), trabalho remunerado (OR: 0,08; IC 95\%: 0,01-0,35; $p=0,0009$ ), não ser procedente da região metropolitana do Recife (OR: 5,83; IC 95\%: 1,53-22,16; $p=0,009$ ), idade gestacional da descoberta da malformação no primeiro e segundo trimestre (OR: 11; IC 95\%: 1,0-120,39; $p=0,049$ ), não possuir partos anteriores (OR: 0,2; IC 95\%: 0,05-0,76; $p=0,01$ ) e abortos anteriores (OR: 4,74; IC 95\%: 1,06$21,16 ; p=0,04)$ e necessitar de tratamento para ansiedade e depressão (OR: 107,3; IC 95\%: 1,94-5915,0; $p=0,02$ ) (Tabela 5).

Por fim, não foi observada boa correlação entre a MFAS com as subescalas de ansiedade $(r=-0,03 ; p=0,79)$ e depressão $(r=-0,33 ; p=0,003)$.

\section{DISCUSSÃO}

Os resultados encontrados por nosso estudo identificaram alta prevalência de sinais e sintomas de ansiedade e depressão, bem como de um médio a alto apego materno-fetal. Corroborando esses resultados, um estudo prévio revelou que $35,3 \%$ das pacientes apresentavam ansiedade moderada e 33,3\%, depressão moderada, e, quanto ao apego, 95,4\% das gestantes com fetos malformados apresentavam máximo apego materno-fetal ${ }^{5}$.

Em relação a essas doenças psiquiátricas, pode-se admitir que a ansiedade advinda da gestação e do processo de enfrentamento do diagnóstico da malformação fetal está interligada com estados depressivos, sendo duas psicopatologias que geralmente coexistem ${ }^{12}$. Essa prevalência revela, assim como em uma pesquisa anterior, que diagnósticos de malformações fetais influenciam negativamente o bem-estar mental das gestantes, mesmo diante de alto apego materno-fetal ${ }^{8}$.

Atrelado a isso, a ausência de religião é um fator de risco para os transtornos psiquiátricos abordados. Autores demonstram que possuir religião atua na defesa contra as doenças psiquiátricas, aumentando a regulação dos circuitos neurais que modulam recompensa e motivação, resposta ao medo, regulação da emoção e comportamento social adaptativo ${ }^{13}$. Assim, a presença de suporte, seja religioso ou social, proporciona experiências menos ameaçadoras, uma vez que oferece mais recursos para o enfrentamento de problemas ${ }^{5}$. Corroborando esses fatos, a literatura demonstra que a diminuição ou a falta de propósito de vida e, principalmente, a baixa espiritualidade são fatores preditores para menor capacidade de resiliência ${ }^{14}$, fatos esses que ratificam os resultados obtidos neste estudo, mesmo em pacientes com fetos malformados.

Antecedentes pessoais maternos de depressão e/ou ansiedade foram fatores associados a sinais e sintomas depressivos ou ansiosos na atual gestação. Essa relação já está consolidada na literatura, demonstrando que indivíduos com transtornos psiquiátricos anteriormente diagnosticados são mais suscetíveis a novos transtornos ou ao agravamento dos preexistentes ${ }^{15}$. No mesmo sentido, um dos principais 
Tabela 3. Variáveis associadas à ansiedade em gestantes com fetos malformados ( $\mathrm{N}=77)$

\begin{tabular}{|c|c|c|c|c|c|c|c|}
\hline \multirow{2}{*}{ Variável } & \multicolumn{4}{|c|}{ Modelo inicial } & \multicolumn{3}{|c|}{ Modelo final } \\
\hline & $\%$ & OR & IC 95\% & $\mathbf{p}$ & $\mathbf{O R}$ & IC 95\% & p \\
\hline \multicolumn{8}{|l|}{ Religião } \\
\hline Sem religião & 66,7 & 2,1 & $0,50-8,91$ & 0,3 & 3,3 & $1,00-10,70$ & 0,04 \\
\hline Com religião & 40,7 & 1,0 & & & 1,0 & & \\
\hline \multicolumn{8}{|l|}{ Raça/cor } \\
\hline Negras & 75,0 & 2,4 & $0,52-11,24$ & 0,25 & & & \\
\hline Não negras & 36,8 & 1,0 & & & & & \\
\hline \multicolumn{8}{|l|}{ Escolaridade } \\
\hline 1-11 anos & 57,1 & 1,7 & $0,48-5,64$ & 0,41 & & & \\
\hline$\geq 12$ anos & 40,8 & 1,0 & & & & & \\
\hline \multicolumn{8}{|l|}{ Abortos anteriores } \\
\hline Zero & 42,6 & 0,3 & $0,05-1,25$ & 0,09 & & & \\
\hline$\geq 1$ & 62,5 & 1,0 & & & & & \\
\hline \multicolumn{8}{|c|}{ Idade gestacional no diagnóstico da malformação } \\
\hline $1^{\circ}$ e $2^{\circ}$ trimestre & 43,5 & 0,5 & $0,06-3,39$ & 0,44 & & & \\
\hline $3^{\circ}$ trimestre & 75,0 & 1,0 & & & & & \\
\hline \multicolumn{8}{|c|}{ Tempo que sabe da malformação } \\
\hline$\geq 10$ semanas & 31,3 & 0,3 & $0,07-1,10$ & 0,06 & 0,3 & $0,09-0,81$ & 0,01 \\
\hline$<10$ semanas & 57,8 & 1,0 & & & 1,0 & & \\
\hline \multicolumn{8}{|l|}{ Gestação múltipla } \\
\hline Sim & 66,7 & 2,6 & $0,42-15,35$ & 0,3 & & & \\
\hline Não & 44,1 & 1,0 & & & & & \\
\hline \multicolumn{8}{|c|}{ Acompanhamento por outra especialidade } \\
\hline Sim & 70,6 & 2,7 & $0,62-11,97$ & 0,18 & & & \\
\hline Não & 40,0 & 1,0 & & & & & \\
\hline \multicolumn{8}{|c|}{ Antecedentes de ansiedade e depressão } \\
\hline Sim & 87,5 & 16,0 & $0,45-565,19$ & 0,12 & 15,2 & $1,61-142,65$ & 0,01 \\
\hline Não & 42,0 & 1,0 & & & 1,0 & & \\
\hline \multicolumn{8}{|c|}{ Tratamento para ansiedade e depressão prévio } \\
\hline Sim & 75,0 & 0,9 & $0,03-22,01$ & 0,96 & & & \\
\hline Não & 43,5 & 1,0 & & & & & \\
\hline \multicolumn{8}{|l|}{ Apego } \\
\hline Médio & 38,1 & 0,5 & $0,16-1,73$ & 0,29 & & & \\
\hline Alto & 57,1 & 1,0 & & & & & \\
\hline
\end{tabular}

Para a análise multivariada da ansiedade, foram excluídas as variáveis idade, companheiro, naturalidade e procedência, idade gestacional na consulta, internada, planejada, empregada, apoio familiar, tipo de malformação, doença materna, incompatibilidade com a vida, prognóstico ruim, tratamento pós-natal, possibilidade de sobreviver grande, renda mensal e número de gestações e partos, por possuírem $p>0,20$.

fatores de risco para depressão gestacional é a história psiquiátrica, principalmente de depressão pregressa ${ }^{7}$. Além disso, estudo sugeriu que a presença de evento de vida estressante (EVE), como gestação com feto malformado, considerado um EVE grave, favorece níveis maiores de estresse, ansiedade e depressão, visto que essas mulheres estão inseridas em um cenário de gravidez de alto risco e expectativas sobre o concepto ${ }^{5,7}$.
Adicionalmente, estudos mostram a relação inversamente proporcional entre o tempo de diagnóstico da malformação fetal e a prevalência de ansiedade e depressão, haja vista que, na primeira consulta após o diagnóstico fetal, 52,4\% das gestantes possuíam algum nível de ansiedade, ao passo que na última consulta 38\% delas mantiveram-se ansiosas ${ }^{16}$. Em concordância, esta pesquisa observou que o conhecimento da malformação fetal há mais de 10 semanas atuou como fator 
Tabela 4. Variáveis associadas à depressão em gestantes com fetos malformados $(\mathrm{N}=77)$

\begin{tabular}{|c|c|c|c|c|c|c|c|}
\hline \multirow{2}{*}{ Variável } & \multicolumn{4}{|c|}{ Modelo inicial } & \multicolumn{3}{|c|}{ Modelo final } \\
\hline & $\%$ & OR & IC 95\% & p & $\mathbf{O R}$ & IC 95\% & p \\
\hline \multicolumn{8}{|l|}{ Religião } \\
\hline Sem religião & 66,7 & 2,8 & $0,56-13,65$ & 0,2 & 6,4 & $1,87-21,86$ & 0,003 \\
\hline Com religião & 30,5 & 1,0 & & & 1,0 & & \\
\hline \multicolumn{8}{|l|}{ Estado civil } \\
\hline Sem companheiro & 53,3 & 1,6 & $0,35-6,98$ & 0,55 & & & \\
\hline Com companheiro & 29,8 & 1,0 & & & & & \\
\hline \multicolumn{8}{|l|}{ Raça/cor } \\
\hline Negras & 65,0 & 4,5 & $0,52-38,7$ & 0,17 & & & \\
\hline Não negras & 29,8 & 1,0 & & & & & \\
\hline \multicolumn{8}{|l|}{ Escolaridade } \\
\hline 1-11 anos & 53,6 & 3,5 & $0,82-14,69$ & 0,08 & & & \\
\hline$\geq 12$ anos & 30,6 & 1,0 & & & & & \\
\hline \multicolumn{8}{|l|}{ Renda mensal } \\
\hline < 1 salário mínimo & 66,7 & 2,6 & $0,45-15,2$ & 0,28 & & & \\
\hline 21 salário mínimo & 31,6 & 1,0 & & & & & \\
\hline \multicolumn{8}{|l|}{ Gestação planejada } \\
\hline Sim & 51,9 & 4,4 & $1,02-18,54$ & 0,04 & & & \\
\hline Não & 32,0 & 1,0 & & & & & \\
\hline \multicolumn{8}{|l|}{ Gestação múltipla } \\
\hline Sim & 66,7 & 5,8 & $0,82-41,33$ & 0,07 & 5,4 & $1,08-26,8$ & 0,03 \\
\hline Não & 35,3 & 1,0 & & & 1,0 & & \\
\hline \multicolumn{8}{|l|}{ Apoio familiar } \\
\hline Sim & 36,1 & 0,1 & $0,004-3,27$ & 0,21 & & & \\
\hline Não & 80,0 & 1,0 & & & & & \\
\hline \multicolumn{8}{|c|}{ Malformação do sistema cardiovascular } \\
\hline Sim & 58,3 & 1,4 & $0,18-10,79$ & 0,74 & & & \\
\hline Não & 35,4 & 1,0 & & & & & \\
\hline \multicolumn{8}{|c|}{ Malformação do sistema geniturinário } \\
\hline Sim & 22,2 & 0,4 & $0,06-2,06$ & 0,25 & & & \\
\hline Não & 44,1 & 1,0 & & & & & \\
\hline \multicolumn{8}{|c|}{ Acompanhamento multiprofissional } \\
\hline Sim & 52,9 & 1,1 & $0,14-8,89$ & 0,89 & & & \\
\hline Não & 35,0 & 1,0 & & & & & \\
\hline \multicolumn{8}{|c|}{ Antecedentes de ansiedade/depressão } \\
\hline Sim & 87,5 & 14,9 & $0,4-549,58$ & 0,14 & 26,7 & $2,88-246,8$ & 0,003 \\
\hline Não & 33,3 & 1,0 & & & 1,0 & & \\
\hline \multicolumn{8}{|c|}{ Tratamento para ansiedade/depressão } \\
\hline Sim & 75,0 & 0,5 & $0,01-11,88$ & 0,64 & & & \\
\hline Não & 34,8 & 1,0 & & & & & \\
\hline
\end{tabular}

Para a análise multivariada de depressão, excluíram-se as variáveis idade, naturalidade e procedência, idade gestacional na consulta, idade gestacional da descoberta da malformação fetal, tempo que sabe da malformação fetal, internada, empregada, doença materna, incompatibilidade com a vida, prognóstico ruim, tratamento pós-natal, possibilidade de sobreviver é grande, apego materno fetal e número de gestações, partos e abortos, por possuírem $p>0,20$.

protetivo para sinais e sintomas de ansiedade, mas essa variável não foi significante estatisticamente para depressão. Sabe-se que, com o decorrer do tempo, a capacidade de adaptação e de aceitação cresce na gestante e faz com que elas se adequem à realidade, havendo, assim, diminuição dos níveis de ansiedade e depressão e aumento de habilidades em cuidar ${ }^{17}$. 
Tabela 5. Variáveis associadas ao apego materno-fetal em gestantes com fetos malformados ( $N=77)$

\begin{tabular}{|c|c|c|c|c|c|c|c|}
\hline \multirow{2}{*}{ Variável } & \multicolumn{4}{|c|}{ Modelo inicial } & \multicolumn{3}{|c|}{ Modelo final } \\
\hline & $\%$ & OR & IC $95 \%$ & $\mathbf{p}$ & $\mathbf{O R}$ & IC 95\% & $\mathbf{p}$ \\
\hline \multicolumn{8}{|l|}{ Escolaridade } \\
\hline 1-11 anos & 42,9 & 0,23 & $0,05-0,95$ & 0,04 & 0,21 & $0,05-0,82$ & 0,02 \\
\hline$\geq 12$ anos & 61,2 & 1,0 & & & 1,0 & & \\
\hline \multicolumn{8}{|l|}{ Empregada } \\
\hline Sim & 36,0 & 0,06 & $0,01-0,31$ & 0,0008 & 0,08 & $0,01-0,35$ & 0,0009 \\
\hline Não & 63,5 & 1,0 & & & & & \\
\hline \multicolumn{8}{|l|}{ Procedente } \\
\hline Outros & 63,9 & 4,8 & $1,13-20,11$ & 0,03 & 5,83 & $1,53-22,16$ & 0,009 \\
\hline Região metropolitana & 46,3 & 1,0 & & & 1,0 & & \\
\hline \multicolumn{8}{|c|}{ Idade da descoberta da malformação fetal } \\
\hline $1^{\circ}$ e $2^{\circ}$ trimestre & 58,0 & 5,9 & $0,49-71,88$ & 0,16 & 11,0 & $1,00-120,39$ & 0,049 \\
\hline $3^{\circ}$ trimestre & 25,0 & 1,0 & & & 1,0 & & \\
\hline \multicolumn{8}{|l|}{ Partos anteriores } \\
\hline 0 & 47,7 & 0,2 & $0,04-0,74$ & 0,01 & 0,2 & $0,05-0,76$ & 0,01 \\
\hline$\geq 1$ & 63,6 & 1,0 & & & 1,0 & & \\
\hline \multicolumn{8}{|l|}{ Abortos anteriores } \\
\hline 0 & 59,0 & 3,8 & $0,83-17,53$ & 0,08 & 4,74 & $1,06-21,16$ & 0,04 \\
\hline$\geq 1$ & 37,5 & 1,0 & & & 1,0 & & \\
\hline \multicolumn{8}{|c|}{ Antecedentes de ansiedade e depressão } \\
\hline $\operatorname{Sim}$ & 75,0 & 1,4 & $0,05-35,51$ & 0,83 & & & \\
\hline Não & 52,2 & 1,0 & & & & & \\
\hline \multicolumn{8}{|c|}{ Tratamento para ansiedade e depressão } \\
\hline Sim & 87,5 & 159,8 & $1,66-15362,72$ & 0,02 & 107,3 & $1,94-5915,0$ & 0,02 \\
\hline Não & 50,7 & 1,0 & & & 1,0 & & \\
\hline \multicolumn{8}{|l|}{ Sinais/sintomas ansiedade } \\
\hline $\operatorname{Sim}$ & 44,4 & 0,5 & $0,14-1,95$ & 0,33 & & & \\
\hline Não & 63,4 & 1,0 & & & & & \\
\hline \multicolumn{8}{|c|}{ Malformação fetal possui tratamento pós-natal } \\
\hline Sim & 59,6 & 3,2 & $0,66-15,19$ & 0,14 & & & \\
\hline Não & 44,0 & 1,0 & & & & & \\
\hline
\end{tabular}

Para a análise multivariada do apego materno-fetal, foram excluídas idade, religião, companheiro, naturalidade, raça, idade gestacional na consulta, tempo que sabe da malformação fetal, internada, gestação planejada ou múltipla, apoio familiar, tipo de malformação, doença materna, incompatibilidade com a vida, prognóstico ruim, possibilidade de sobreviver é grande, acompanhamento por outra especialidade, subescala de depressão, renda e número de gestações anteriores, por possuírem $p>0,20$.

É evidente ainda que a gestação promove condições especiais que acarretam mudanças nos processos metabólicos e fisiológicos da mulher, culminando em um risco para a mãe e/ou para o feto ${ }^{18}$. Sendo assim, a ocorrência de gestação múltipla foi um dos fatores associados ao desenvolvimento de depressão em nosso estudo. Sabe-se que, diante de uma gestação gemelar associada à malformação fetal, há aumento significativo do risco gestacional, seguido de alta predisposição ao desenvolvimento de alterações psíquicas ${ }^{18}$, além da preocupação com a saúde do feto sem alterações morfológicas fetais. Essa condição de maior vulnerabilidade acarreta sentimentos como infelicidade, estresse e preocupações quanto à gestação, que, associados à dificuldade de adaptação da mulher, favorecem o desenvolvimento da depressão na gravidez ${ }^{19}$.

Em relação ao apego materno-fetal, este estudo não relatou associação clara entre a presença de ansiedade e depressão e a alteração no apego entre a gestante e seu filho. Isso porque os resultados encontrados por nós demonstraram que, independentemente do grau do transtorno psiquiátrico identificado na gestante, a maioria delas apresentou alto apego em relação ao seu filho. Achados literários de estudos que analisaram essa relação ratificam nossos resultados, demonstrando que $90 \%$ das gestantes analisadas, com fetos malformados, apresentaram um alto apego, apesar da presença de ansiedade e/ou depressão diagnosticada ${ }^{17}$. 
Em outro contexto, quando foram avaliados os fatores associados a um menor e médio apego materno-fetal, comparado a alto apego, observou-se que a escolaridade das gestantes entre 1 e 11 anos esteve mais vulnerável ao alto apego materno-fetal, em comparação com níveis de escolaridade superiores a 12 anos. Porém, outros autores afirmam que o alto grau de escolaridade está relacionado à priorização do enfrentamento focalizado no problema, estratégia essa que fortalece o vínculo materno-fetal ${ }^{2,20}$. A divergência entre esses resultados pode estar relacionada ao pequeno número amostral, que, em sua maioria, foi composto por gestantes com escolaridade até 12 anos.

Atrelado a isso, é evidente que contextos de crise econômica, baixas condições socioeconômicas e instabilidade profissional são variáveis que contribuem para a dificuldade de adaptação materna e familiar à gestação, uma vez que a gravidez necessita de reestruturação em várias dimensões ${ }^{17,21}$. Juntamente com a preocupação econômica, o impacto da notícia de uma malformação fetal possivelmente transforma essa mudança em uma crise emocional, favorecendo sentimentos de inferioridade e baixa autoestima materna, além de diminuir a responsividade psicossocial, afetando negativamente o vínculo materno-fetal em longo prazo ${ }^{5,6}$. Seguindo essa perspectiva, observou-se que estar empregada profissionalmente durante o diagnóstico fetal foi fator protetor para apego médio.

Por outro lado, as gestantes que não residiam no Recife e região metropolitana (RMR) apresentaram-se como de risco para escores médios de apego materno-fetal. É evidente que a distância física entre a residência da paciente e o serviço de saúde desempenha influência na adesão às condutas e ao tratamento ${ }^{22}$. No entanto, poucas pesquisas investigaram o impacto da distância física ao hospital sobre o apego materno-fetal. Acredita-se que, como o estudo foi realizado na cidade do Recife, agregado à prevalência de baixa renda, as gestantes que não residiam na RMR tiveram mais dificuldade em manter o acompanhamento corretamente com o setor de medicina fetal, localizado na cidade do Recife, Pernambuco. Como consequência, há um déficit de informações sobre a gestação, o que afeta a dinâmica mãe-filho e dificulta o enfrentamento de possíveis complicações ${ }^{20}$.

Nesse contexto, foi evidente, ainda, que o período do diagnóstico da malformação teve influência no apego materno-fetal. O diagnóstico da malformação fetal durante o primeiro e o segundo trimestre, por exemplo, foi fator de risco para o médio apego. Isso porque estudos mostram que a notícia de malformação fetal nos dois primeiros trimestres eleva os níveis de estresse e ansiedade materna e, consequentemente, diminui o apego com o feto ${ }^{23,24}$. Entretanto, outros dados mostram que quanto mais precoce o conhecimento sobre a malformação fetal, maior tempo a família tem para a criação de estratégias de aceitação e enfrentamento da situação ${ }^{25}$. No entanto, como o segundo trimestre é o período fortalecedor do vínculo materno-fetal, devido à presença de movimentos fetais acentuados, espera-se elevado apego. Porém, as gestantes que recebem diagnóstico de anomalias fetais nesse período tendem a não responder às expectativas ${ }^{26}$.

Em contraponto, a nuliparidade foi fator protetor para apego médio, o que reflete em multíparas como grupo de risco. Isso pode ser explicado pelo fato de que as nulíparas acabam por criar maiores expectativas em relação à gestação, quando comparada às multíparas ${ }^{27}$. Aliado a isso, é evidente que a gravidez, principalmente do segundo ou terceiro filho, pode causar muita ansiedade devido às mudanças no sistema familiar já equilibrado, por isso achados literários evidenciam que as gestantes multíparas tendem a apresentar redução do apego em uma nova gestação ${ }^{28}$.

Ademais, vivenciar um aborto pode alterar a saúde mental das mulheres em pequeno ou longo prazo, podendo até mesmo persistir no curso de gestações subsequentes e mudar o sentimento em relação ao filho que está por vir, diminuindo o apego materno-fetal ${ }^{29,30}$. Contrapondo-se ao esperado, evidenciamos menor apego materno-fetal na ausência de antecedentes de aborto. Justifica-se pelo fato que nosso estudo foi realizado em fetos malformados, o que pode ter contribuído para que gestantes com aborto prévio tenham alto apego, devido ao pensamento materno sobre necessidade dos cuidados ao recém-nascido com malformação.

Em contrapartida, ter realizado tratamento prévio para ansiedade e/ou depressão foi preditivo para nível médio de apego. Esse achado vai ao encontro com a literatura, pois, como a depressão é uma condição crônica e recorrente, o não tratamento adequado implica seu prolongamento e, diante de uma gestação de alto risco, essa psicopatologia tende a ser exacerbada, implicando a díade mãe-feto 7,17. Destaca-se que, entre a parcela estudada, a frequência de antecedentes de ansiedade e/ou depressão foi igual à realização de tratamento pregresso, porém, como limitação, esta pesquisa não avaliou a efetividade e a qualidade desse tratamento anterior.

Por fim, não foi observada boa correlação entre a MFAS e as subescalas de ansiedade $(r=-0,03)$ e depressão ( $r=$ -0,33). Como nosso estudo abordou um grupo específico de gestantes de alto risco, ou seja, com fetos malformados, acredita-se que a presença de sinais e sintomas clínicos de ansiedade e depressão seja decorrente da notícia de malformação, da incerteza do prognóstico e devido às demais variáveis analisadas. Entretanto, entende-se que não necessariamente essas psicopatologias irão influenciar negativamente no apego da mãe com o feto. No mesmo sentido, um estudo prévio com gestantes de baixo risco não encontrou associação significativa entre sinais e sintomas de ansiedade e depressão e o grau de apego materno-fetal ${ }^{13,32}$, tornando 
essa não associação plausível, tendo em vista a concordância do resultado quando analisadas gestantes com e sem malformações fetais.

Vale ressaltar que o presente estudo não é isento de limitações, considerando seu desenho transversal, logo não foi possível determinar a causalidade temporal entre o apego materno-fetal, os sintomas psiquiátricos e a presença de malformação fetal nas gestantes. Diante disso, sugere-se a realização de novas pesquisas que abordem esses aspectos por meio de diferentes desenhos de estudo. Outro ponto está na utilização de escalas de autopreenchimento, pois elas podem ser cabíveis de erros de interpretação por parte das participantes, além de apresentarem viés de memória. Contudo, salienta-se que os autores ficaram disponíveis para retirada de qualquer dúvida por parte das integrantes durante todo o processo, minimizando, assim, os possíveis erros.

Um ponto forte e que merece destaque do estudo foi o tamanho amostral, haja vista que os estudos prévios, em geral, apontam para uma população total mais reduzida, ainda mais por ser tratar de um grupo delicado.

\section{CONCLUSÃO}

Diante dos dados analisados, percebe-se que as gestantes que possuem fetos diagnosticados com alguma malformação tendem a desenvolver ansiedade e/ou depressão com maior prevalência, apesar do médio e alto apego materno-fetal. Salienta-se, assim, a importância de programas específicos para rastreio e assistência à saúde mental dessas muIheres durante o período pré-natal, a fim de encorajá-las a serem ativas no processo de enfrentamento e na tomada de decisões relativas ao binômio mãe-filho.

\section{CONTRIBUIÇÕES INDIVIDUAIS}

Gustavo Fonseca de Albuquerque Souza - (1) Contribuiu significativamente na concepção e desenho dos estudos ou na análise e interpretação dos dados; (2) contribuiu substancialmente na elaboração do artigo ou revisando criticamente o seu conteúdo intelectual; (3) aprovou a versão final a ser publicada.

Alex Sandro Rolland Souza - (1) Contribuiu significativamente na concepção e desenho dos estudos ou na análise e interpretação dos dados; (2) contribuiu substancialmente na elaboração do artigo ou revisando criticamente o seu conteúdo intelectual; (3) aprovou a versão final a ser publicada.

Gabriella de Almeida Figueredo Praciano - (1) Contribuiu significativamente na análise e interpretação dos dados; (2) contribuiu substancialmente na elaboração do artigo; (3) aprovou a versão final a ser publicada.
Esther Soraya Lima de França - (1) Contribuiu significativamente na análise e interpretação dos dados; (2) contribuiu substancialmente na elaboração do artigo; (3) aprovou a versão final a ser publicada.

Cinthia Freire Carvalho - (1) Contribuiu significativamente na análise e interpretação dos dados; (2) contribuiu substancialmente na elaboração do artigo; (3) aprovou a versão final a ser publicada.

Sérgio de Sá Leitão Paiva Júnior - (1) Contribuiu significativamente na análise e interpretação dos dados; (2) contribuiu substancialmente na elaboração do artigo; (3) aprovou a versão final a ser publicada.

Manuela Barbosa Rodrigues de Souza - (1) Contribuiu significativamente na concepção e desenho dos estudos ou na análise e interpretação dos dados; (2) contribuiu substancialmente na elaboração do artigo ou revisando criticamente o seu conteúdo intelectual; (3) aprovou a versão final a ser publicada.

Nadja Maria Jorge Asano - (1) Contribuiu significativamente na concepção e desenho dos estudos ou na análise e interpretação dos dados; (2) contribuiu substancialmente na elaboração do artigo ou revisando criticamente o seu conteúdo intelectual; (3) aprovou a versão final a ser publicada.

\section{CONFLITO DE INTERESSES}

Declaramos que não há conflito de interesses entre os autores deste artigo.

\section{REFERÊNCIAS}

1. Borges DA, Ferreira FR, Mariutti MG, Almeida DA. A depressão na gestação: uma revisão bibliográfica. Revista de Iniciação Científica da Libertas. 2011;1(1):85-99.

2. Borges MM, Petean EBL. Malformação fetal: enfrentamento materno, apego e indicadores de ansiedade e depressão. Rev SPAGESP. 2018;19(2):137-48.

3. Piccinini CA, Gomes AG, Moreira LE, Lopes RS. Expectativas e sentimentos da gestante em relação ao seu bebê. Psic Teor Pesq. 2004;20(3):223-32.

4. Cranley MS. Desenvolvimento de uma ferramenta para a medição do apego materno durante a gravidez. Nurs Res. 1981;30(5):281-4.

5. Vasconcelos L, Petean EBL. 0 impacto da malformação fetal: indicadores afetivos e estratégias de enfrentamento das gestantes. Psic Saúde Doenças. 2009;10(1):69-82.

6. Antunes MSC, Patrocinio C. A malformação do bebé: vivências psicológicas do casal. Psic Saúde Doenças. 2007;8(2):239-51.

7. Barros VC, Santos JF, Lima LA, Fonseca DL, Lovisi GM. Depressão e apoio social em gestantes de fetos com malformações atendidas em um hospital materno-infantil público de referência no Rio de Janeiro. Cad Saúde Coletiva. 2013;21(4):391-402.

8. Asplin N, Wessel H, Marions L, Ohman SG. Maternal emotional wellbeing over time and attachment to the fetus when a malformation is detected. Sex Reprod Healthc. 2015;6(3):191-5.

9. Feijó MC. Validação brasileira da Maternal-Fetal Attachment Scale. Arq Bras Psicol. 1999:51(4):52-62.

10. Botega NJ, Bio MR, Zomignani MA, Garcia C, Pereira WAB. Transtornos de Humor em Enfermaria de Clínica Médica e Validação de Escala de Medida (HAD) de Ansiedade e Depressão. Rev Saúde Pública. 1995;29(5):355-63. 
11. Dancey (P, Reidy J. Estatística sem matemática para psicologia. Porto Alegre: Artmed; 2006

12. Adouard F, Glangeaud-Freudenthal NM, Golse B. Womens mental health validation of the Edinburgh postnatal depression scale (EPDS) in a sample of women with highrisk pregnancies in France. Arch Womens Mental Health. 2005;8(2):89-95.

13. Southwick SM, Charney DS. The science of resilience: implications for the prevention and treatment of depression. Science. 2012;338(6103):79-82.

14. Min J, Jung Y, Kim D. Características associadas à baixa resiliência em pacientes com depressão e/ou transtornos de ansiedade. Qual Life Res. 2013;22:231-41.

15. Jacobson N, Newman M. Anxiety and depression as bidirectional risk factors for one another: A meta-analysis of longitudinal studies. Psychol Bull. 2017;143(11):1155-200.

16. Titapant V, Chuenwattana P. Psychological effects of fetal diagnoses of non-lethal congenital anomalies on the experience of pregnant women during the remainder of their pregnancy. J Obstet Gynaecol Res. 2015;41(1):77-83.

17. Borges MM, Pinto MJC, Mos Vaz DC. Apego materno-fetal e enfrentamento de gestantes frente ao diagnóstico de malformação. Arq Ciênc Saúde. 2015;22(2):27-32.

18. Santos DTA, Campos CSM, Duarte ML. Perfil das patologias prevalentes na gestação de alto risco em uma maternidade escola de Maceió, Alagoas, Brasil. RBMFC. 2014;9(30):13-22.

19. Silva MMJ, Lima GS, Monteiro JCS. Depression in pregnancy: risk factors associated with its occurrence. Rev Eletrônica Saúde Mental Álcool Drog. 2020;16(1):1-12.

20. Benute GRG, Nonnenmacher D, Evangelista LFM, Lopes ML, Souza MC, Zugaib M. Cardiopatia fetal e estratégias de enfrentamento. Rev Bras Ginecol Obstet. 2011;33(9):227-33.

21. Perossa GB, Silveira FCP, Canavez IC. Ansiedade e depressão de mães de recém-nascidos com malformações visíveis. Psic Teor Pesq. 2008;24(1):29-35.
22. Amram 0, Shoveller J, Hogg R, Wang L, Sereda P, Barrios R, et al. Distance to HIV care and treatment adherence: Adjusting for socio-demographic and geographical heterogeneity. Spat Spatiotemporal Epidemiol. 2018;27:29-35.

23. Fontoura FC, Cardoso MVL, Rodrigues SE, Almeida PC, Carvalho LB. Anxiety of mothers of newborns with congenital malformations in the pre- and postnatal periods. Rev Lat Am Enfermagem. 2018;24(14):e3080

24. Kaasen A, Helbig A, Malt U, Naes T, Skari H, Haugen G. Maternal psychological responses during pregnancy after ultrasonographic detection of structural fetal anomalies: A prospective longitudinal observational study. PLoS One. 2017;12(3):e0174412.

25. Sousa S. A saúde do feto. In: Sá E, editor. Psicologia do feto e do bebê. Lisboa: Fim de Século; 2003.

26. Saviani-Zeoti F, Petean EBL. Apego materno-fetal, ansiedade e depressão em gestantes com gravidez normal e de risco: estudo comparativo. Estud Psicol. 2015;32(4):675-83.

27. Nichols M, Roux G, Harris N. Primigravid and multigravid women: Prenatal perspectives. J Perinat Educ. 2007;16(2):21-32.

28. Fernandes RB, Silveira RA, Marques A. Formação do vínculo materno-fetal e a influência da assistência pré-natal. Enciclopédia Biosfera. 2017;14(26):1142.

29. Toffol E, Koponen P, Partonen T. Miscarriage and mental health: results of two populationbased studies. Psychiatry Res. 2013;205(1-2):151-8.

30. Lok IH, Yip ASK, Lee DTS, Sahota D, Chung TKH. A 1-year longitudinal study of psychological morbidity after miscarriage. Fertil Steril. 2010;93(6):1966-75.

31. Zeferino MGM, Furegato ARF. Aborto, depressão, autoestima e resiliência: uma revisão. Saúde Transf Soc. 2013;4(3):115-9.

32. Schmidt EB, Argimon IIL. Vinculação da gestante e apego materno fetal. Paidéia. 2009;19(43):211-20 